\title{
Little brainiacs and big dummies: Are we selecting for stupid, stout, or small dogs?
}

\author{
Mark Rishniw* and Curtis W. Dewey \\ Department of Clinical Sciences, College of Veterinary Medicine, Cornell University, Ithaca, NY, 14853, USA
}

\begin{abstract}
Background: Brain size has been associated with intelligence of various orders and families of animals, leading to the concept of encephalization. Brain size scales with body weight between species within mammals to approximately the 0.67 power. However, within species, this scaling exponent appears to be much smaller (approximately 0.27 power). Aim: We examined whether this relationship has persisted in dogs over the 120 years since this was originally observed. Methods: Comparative cross-sectional study of magnetic resonance imaging (MRI) data obtained from 127 dogs, compared to historical data from 157 dogs and 24 non-dog canid species.

Results: Brain size in dogs measured by MRI had a scaling exponent virtually identical to that observed previously (0.24 vs. 0.26). However, the proportionality constant was smaller, suggesting that dogs in the study cohort had relatively smaller brains than the historical cohort. Absolute brain size appeared to have both a lower and upper limit in dogs. When compared to non-dogs canids, the most appropriate "representative" size for a "typical dog" when examining allometric scaling across Canidae appeared to be approximately $10-15 \mathrm{~kg}$.

Conclusions: We interpreted the slight reduction in relative brain size to be a function of increased obesity in the study cohort compared to dogs examined 120 years ago. Further, we suggest that dog brains have a finite lower size limit. Finally, concepts of encephalization should not be applied to dogs.
\end{abstract}

Keywords: Brain size, Canine, Encephalization.

\section{Introduction}

Brain size in mammals is related to body size by a nonlinear relationship. Most researchers have accepted that, across species, the relationship fits a power function with an exponent ranging from 0.56 to 0.67 , first identified over 100 years ago (Dubois, 1897; Lapicque, 1898), such that smaller mammals have a larger proportional brain size than larger mammals. Exceptions to this general relationship exist, which led initially to the development of the "encephalization index" (Dubois, 1897; Stephan and Andy, 1969) or "encephalization quotient" (Jerison, 1973). This index or quotient putatively estimates "evolved intelligence" or other behavioral traits, under the assumption that the bigger the brain per kilogram of bodyweight beyond what would be required for basic neural functions, the greater the intelligence. Numerous studies attempt to relate relative brain size (or encephalization) to various attributes of a species, including intelligence (Roth and Dicke, 2005), metabolic demand ( $\mathrm{McNab}$ and Eisenberg, 1989; Heymsfield et al., 2009), sociality (Pérez-Barbería et al., 2007; Dunbar and Bever, 2010; Shultz and Dunbar, 2010), and gestation time (Sacher and Staffeldt, 1974).

Gittleman (1986) showed that species of the family Canidae adhere to the inter-species relationship of brain size to body size, with a scaling exponent of approximately 0.64 . However, Louis Lapicque had first observed that intra-species relationships, at least in dogs, scale with a much smaller exponent of 0.24 (Lapicque, 1898), using a dataset provided by Charles Richet (Richet, 1891). Bronson confirmed this relationship, with a scaling exponent of 0.27 , suggesting that breed, rather than bodyweight, might determine intelligence within a particular species (Bronson, 1979). A smaller scaling exponent would produce a much smaller difference in brain size between dogs of different sizes than between species spanning the same bodyweight range. Jerison noted, but did not reference or provide evidence, that the brain-size-tobody-size relationship between species differs from that observed within species, also suggesting a power function approximating 0.25 , rather than 0.67 (Jerison, 1973, 1977). His claims appear to be unwarranted extrapolations of the study of Lapicque from dogs to all species.

However, not all inter-species brain-bodyweight relationships adhere to the general scaling law $\left(\mathrm{BW}^{0.67}\right)$. Sholl examined the relationship in species of Macaques, and found a very flat relationship between species of the genus Macaca, with a scaling exponent of 0.18 (Sholl, 1948).

Dogs provide a unique opportunity to study the intraspecies relationship between brain size and body size, because they vary in size more than any other species (approximately 70 -fold differences), ranging from 
an adult weight of $<1 \mathrm{~kg}$ for some Yorkshire terriers to $>70 \mathrm{~kg}$ for Irish Wolfhounds and St. Bernards. Deriving intra-species relationships in most species is problematic because the weight range tends to be somewhat restricted, resulting in a lack of sufficient resolution to determine a true relationship. We could find no data examining intra-species relationships in other species of mammals.

We had the opportunity to measure brain volume in live dogs using magnetic resonance imaging (MRI). Because brain volume closely approximates brain weight, we could calculate the brain-size-to-body-size ratio. We then calculated each dog's "encephalization quotient" and compared our data to those of Richet, obtained 130 years ago.

\section{Materials and Methods}

We obtained bodyweight, breed, and forebrain volume data for dogs from three sources: dogs being evaluated by MRI for various neurological problems, or that had been imaged as healthy controls for other studies; dogs originally measured by Richet (1891); and data examining the family Canidae (Gittleman, 1986).

Details of the imaging of our dataset have been previously published (Estey et al., 2017). We measured brain volumes using Materialize Mimics software.

To make direct comparisons with the Richet data, we calculated brain weight from our volumetric data using the following equation:

$$
\text { Mass }=1.04 \times \text { volume }
$$

where 1.04 is the density of brain tissue.

Because Richet measured brain weight using formalinfixed samples, we adjusted for the effect of formalin using the following equation:

$$
\begin{aligned}
\text { Brain weight }= & \text { Brain weight }_{\text {formalin }}-(\text { Brain weight } \\
& \times 0.038)(\text { Fox }, 1996) .
\end{aligned}
$$

We used the data provided by Gittleman as the relevant inter-species cohort (Gittleman, 1986).

We then plotted brain weights against body weights, and fitted these plots, first as raw data and then after $\log -\log$ transformation, to a power function for all three datasets.

To examine whether the slopes and intercepts of the brain-weight-to-body-weight relationships have changed over that last 100 years, we compared the slopes and intercepts between the Richet dataset and our dataset using regression analysis and Analysis of Variance. We used commercial statistical software for all analyses [MedCalc Statistical Software version 19.0.7 (MedCalc Software bvba, Ostend, Belgium; https://www.medcalc.org; 2019)].

\section{Results}

We included 127 dogs with imaging data, 157 dogs from the Richet dataset and 24 species belonging to the family Canidae from the Gittleman study. Dogs in our cohort ranged in body weight from $1.3 \mathrm{~kg}$ (Yorkshire terrier) to $79 \mathrm{~kg}$ (St. Bernard). Dogs from the Richet dataset ranged in body weight from 1.25 to $43.5 \mathrm{~kg}$. Canids from the Gittleman dataset ranged in body weight from $1.5 \mathrm{~kg}$ (Fennec fox) to $33 \mathrm{~kg}$ (wolf).

Dogs from our dataset and those from Richet showed almost identical scaling exponents (0.26 vs. 0.24, $p$ $=0.26$ ), and considerably different from canids in general (0.65) (Fig. 1). Surprisingly, dogs from the Richet dataset had brains approximately $10 \mathrm{~g}$ larger per unit body weight than dogs from our dataset (Fig. 1), resulting in different intercepts of the two, but virtually parallel, regression slopes $(p<0.00001)$.

The relatively small scaling exponent for dogs $(0.26)$ resulted in encephalization quotients ranging from 4 for the smallest dogs, to 0.5 for the largest dogs (Fig. 2). Using this approach, small dogs had higher encephalization quotients than their non-dog canid counterparts of comparable size, but large dogs had lower encephalization quotients than similarly sized non-dog canids.

\section{Discussion}

Our data confirm the original observations of Richet and Lapicque, demonstrating that domestic dogs have a brain-size-to-body-size relationship that differs dramatically from the canonical inter-species relationship, best fitting a power function of 0.26 rather than 0.65-0.67. However, modern dogs had lower relative brain sizes than dogs examined a century ago.

Why do dogs today have smaller relative brain size than dogs 100 years ago?

There are multiple possible explanations, both biological and methodological, for this observation.

The first biological explanation that could account for our observation is that humans have selectively bred dogs with smaller brains. However, breeders rarely, if ever, select specifically for "small cranial cavities or brain volume" or even small heads. Furthermore, although the decrease was small (about $10 \mathrm{~g}$ ), a one-hundred-year time period would be unlikely to dramatically affect brain size from an evolutionary perspective.

A more reasonable biological explanation is that modern dogs are fatter than their century-old counterparts. Neither dataset recorded body condition scores, but plentiful evidence exists that dogs, like people, in the United States are experiencing an increasing prevalence of obesity (Banfield Pet Hospital, 2012; Chandler etw wwal., 2017). Consequently, a dog of a particular size today would likely be somewhat lighter a century ago. Researchers a century ago recognized the importance of examining brain-size-to-body-size relationships in animals that are in "optimal condition," and cautioned including data for domestic animals that often differ in body condition from their wild counterparts.

Methodological explanations include sample population differences (although both datasets covered 


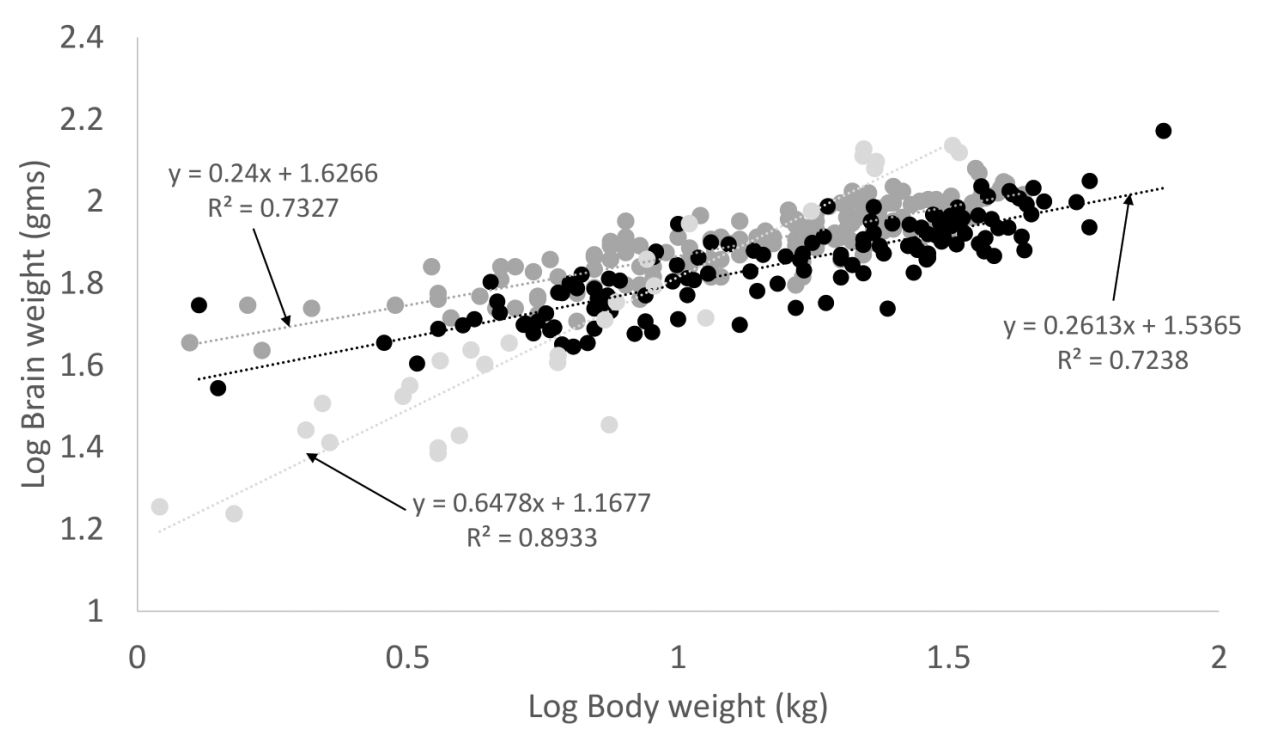

Fig. 1. Brain weight scales with body weight to the 0.26 power in dogs. The black dots and line represent domestic dogs from the current study, dark gray dots and line represent domestic dogs from the Richet dataset, and the light gray dots and line represent canids from the Gittleman dataset.

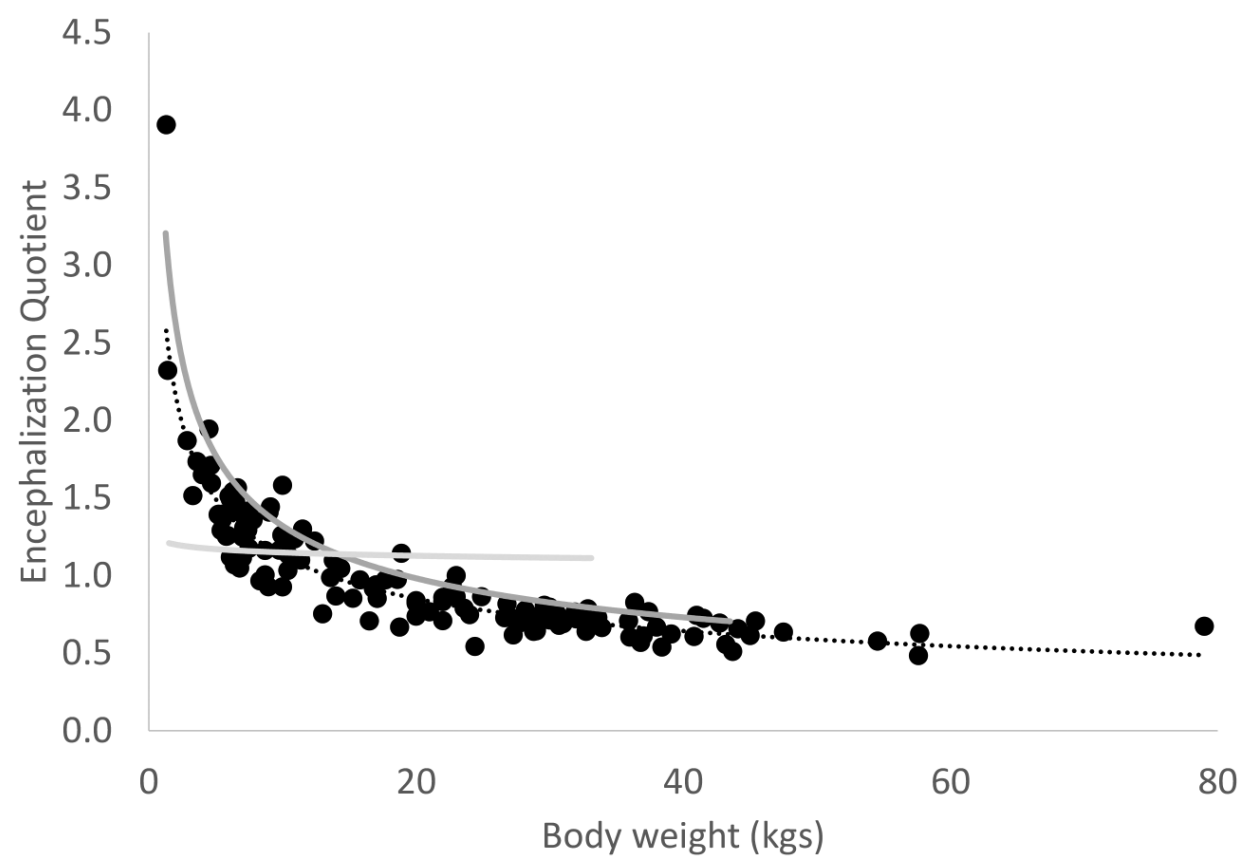

Fig. 2. Encephalization quotient decreases with increasing body weight in dogs. The dashed black line represents the regression line for dogs, the dark gray solid line represents the regression line for dogs from the Richet dataset, and the light gray solid line represents the regression line for canids from the Gittleman dataset. The regression line for canids crosses the regression lines for dogs at approximately $10-15 \mathrm{~kg}$.

similar body weight ranges, and a variety of breeds), and the assumptions made in converting volume to mass (or adjusting for fixation).

Are little dogs smarter than big dogs?

No behavioral evidence exists that small breed dogs are more intelligent than large breed dogs. Indeed, common wisdom suggests the opposite- that small breeds are less "trainable," less sociable, and less smart than large breed dogs (Helton, 2010; Helton and Helton, 2010; Jakovcevic et al., 2010). One study suggested that large breeds demonstrated higher social cognition scores than small breeds, based on a visual pointing cue 
test (Helton and Helton, 2010). A more recent study supported this observation (Horschler et al., 2019), but another investigator challenged the methodology of this study (Montgomery, 2019). Furthermore, other investigators failed to observe a relationship between brain size and cognition (Broadway et al., 2017). On the other hand, researchers identified a spectrum of cognitive abilities in a single working breed (Arden and Adams, 2016). Many of the differences identified in these cognitive studies might simply be identifying selection of specific morphotypes for specific tasks, and certain cognitive skills that underpin those tasks, rather than selecting for or against overall intelligence. Such a hypothesis finds support in studies that identify "trainability" with working breeds vs. non-working breeds (Mehrkam and Wynne, 2014; Eken Asp et al., 2015), rather than size. Given that working breeds are generally larger than non-working breeds, size might simply be a poor surrogate for "working breed."

This observation also flies in the face of assigning an encephalization quotient to dogs - in our study, small breed dogs had a relative brain size far exceeding their "expected" brain size, while large and giant breed dogs had relative brain sizes of mental midgets. Therefore, applying an encephalization quotient to dog breeds appears nonsensical, and challenges the entire anthropocentric notion of encephalization as a measure of intelligence.

\section{What is the appropriate encephalization quotient for} domestic dogs?

Assigning a single encephalization quotient to Canis canis (Canis familiaris) seems similarly problematicwhich quotient (from 4.0 to 0.5 ) would be most appropriate? Creating an "average" encephalization quotient depends on the sample population from which the average is obtained, and is, therefore, prone to error. Others have suggested that the domestic dog has an encephalization quotient that is substantially lower than wolves, arguing that domestication has led to a decrease in "intelligence." However, such claims are based on examining dogs that had a weight similar to that of wolves $(30 \mathrm{~kg})$.

One way to approach this question is to examine the intersection of the curves for domestic dogs and all canids. These lines cross at a weight of approximately $10 \mathrm{~kg}$. Could this intersection be considered the "prototypical weight" of the original, domesticated dog? Examination of stray dogs in areas where little or no breed selection pressure exists, suggests that this might indeed be a reasonable estimate for prototypical dogs, which some have estimated to weigh between 9 and $15 \mathrm{~kg}$ (Coppinger and Coppinger, 2001). These dogs are generally reasonably small and thin (importantly, they are not overweight or obese). From our data, dogs ranging from 9 to $11 \mathrm{~kg}$ had an average encephalization quotient of 1.2. This would be no different from the encephalization of other canids, including the wolf and fox.
What makes a dog a dog?

Our data, and those of Richet, provide some interesting limits to brain size (Richet, 1891). Dogs as small as $1.3 \mathrm{~kg}$ have brains that are approximately $40-50 \mathrm{~g}$, while dogs 30-40 times that size have brains that rarely exceed $110 \mathrm{~g}$. This relatively small increase in brain size, and an absolute apparent lower limit, which is considerably greater than comparably sized mammals of other species, suggests that, regardless of how small domestic dogs become, brain size must be conserved to accommodate sufficient neuronal complexity for the dog to maintain its "dogness." Similarly, because large and giant-breed dogs do not behave or socialize differently from small breed dogs, they do not require additional cognitive functions.

\section{Does a lower brain limit result in developmental problems in small dogs?}

If absolute brain size is limited in dogs, attempting to reduce body size could have clinical consequences. Open fontanelles, where cranial growth plates on top of the skull fail to close, are almost exclusively seen in toy and miniature breeds. These breeds have relative macrocrania, often with a domed large skull and a relatively small face. These breeds are also predisposed to hydrocephaly. Such cranial phenotypes might result from attempting to fit a brain required for a dog to maintain its "dogness" into an ever-shrinking canine head.

\section{Conclusion}

Our data confirm the observations made over 130 years ago by Richet, and the subsequent analysis of Richet's data by Lapicque. However, our data suggest that the dogs of the 21st century are heavier (fatter) than their counterparts from a century ago. Finally, our data suggest that the theory of encephalization does not apply within species, and is largely constant within a genus if an appropriate representative weight is selected.

\section{Supplemental data}

The full dataset can be obtained at this link (https:// www.openveterinaryjournal.com/brain-volumesovj-2021).

\section{Conflict of interest}

The authors have no conflicts of interest to declare. Author contributions

Mark Rishniw conceived of the study, analyzed the data, and drafted the manuscript. Curtis Dewey acquired the MRI data, consulted on the analysis, and edited the manuscript.

\section{References}

Arden, R. and Adams, M.J. 2016. A general intelligence factor in dogs. Intelligence 55, 79-85.

Banfield Pet Hospital. 2012. Dog and cat diseases banfield pet hospital ${ }^{\circledR}$ State of Pet Health 2012 Report. Available via https://www.banfield.com/ Banfield/media/PDF/Downloads/soph/BanfieldState-of-Pet-Health-Report_2012.pdf (Accessed 16 August 2019). 
Broadway, M.S., Samuelson, M.M., Christopher, J.L., Jett, S.E. and Lyn, H. 2017. Does size really matter? Investigating cognitive differences in spatial memory ability based on size in domestic dogs. Behav. Processes 138, 7-14.

Bronson, R.T. 1979. Brain weight-body weight scaling in breeds of dogs and cats. Brain. Behav. Evol. 16, 227-236.

Chandler, M., Cunningham, S., Lund, E.M., Khanna, C., Naramore, R., Patel, A. and Day, M.J. 2017. Obesity and associated comorbidities in people and companion animals: a one health perspective. J. Comp. Pathol. 156, 296-309.

Coppinger, R. and Coppinger, L. 2001. Chapter 2: village dogs, in: Dogs : a startling new understanding of canine origin, behavior, and evolution. New York, NY: Scribner, pp: 69-85.

Dubois, E. 1897. Sur le rapport du poids de l'encéphale avec la grandeur du corps chez les mammifères. Bull. la Société d'anthropologie Paris. 8, 337-376.

Dunbar, R.I.M. and Bever, J. 2010. Neocortex size predicts group size in carnivores and some insectivores. Ethology. 104, 695-708.

Eken Asp, H., Fikse, W.F., Nilsson, K. and Strandberg, E. 2015. Breed differences in everyday behaviour of dogs. Appl. Anim. Behav. Sci. 169, 69-77.

Estey, C.M., Dewey, C.W., Rishniw, M., Lin, D.M., Bouma, J., Sackman, J. and Burkland, E. 2017. A subset of dogs with presumptive idiopathic epilepsy show hippocampal asymmetry: a volumetric comparison with non-epileptic dogs using MRI. Front. Vet. Sci. 4, 183.

Fox, M. 1966. Further observations on age differences in the effects of formalin on the canine brain in vitro. Experientia 22, 447-448.

Gittleman, J.L. 1986. Carnivore brain size, behavioral ecology, and phylogeny. J. Mammal. 67, 23-36.

Helton, W.S. 2010. Does perceived trainability of dog (Canis lupus familiaris) breeds reflect differences in learning or differences in physical ability? Behav. Processes 83, 315-323.

Helton, W.S. and Helton, N.D. 2010. Physical size matters in the domestic dog's (Canis lupus familiaris) ability to use human pointing cues. Behav. Processes 85, 77-79.

Heymsfield, S.B., Chirachariyavej, T., Rhyu, I.J., Roongpisuthipong, C., Heo, M. and Pietrobelli, A. 2009. Differences between brain mass and body weight scaling to height: potential mechanism of reduced mass-specific resting energy expenditure of taller adults. J. Appl. Physiol. 106, 40-48.

Horschler, D.J., Hare, B., Call, J., Kaminski, J., Miklósi, Á. and MacLean, E.L. 2019. Absolute brain size predicts dog breed differences in executive function. Anim. Cogn. 22, 187-198.
Jakovcevic, A., Elgier, A.M., Mustaca, A.E. and Bentosela, M. 2010. Breed differences in dogs' (Canis familiaris) gaze to the human face. Behav. Processes 84, 602-607.

Jerison, H.J. 1973. Gross brain indices and the meaning of brain size, in: evolution of the brain and intelligence. New York, NY: Academic Press, pp: 55-81.

Jerison, H.J. 1977. The theory of encephalization. Ann. N. Y. Acad. Sci. 299, 146-160.

Lapicque, L. 1898. Sur le rapport du poids de l'encéphale avec la grandeur du corps chez les mammifères. In Comptes Rendus Des Séances de La Société de Biologie et de Ses Filiales. Ed., Maretheux, L. Paris, France: Masson, pp: 62-63.

McNab, B.K. and Eisenberg, J.F. 1989. Brain size and its relation to the rate of metabolism in mammals on JSTOR. Am. Nat. 133, 157-167.

Mehrkam, L.R. and Wynne, C.D.L. 2014. Behavioral differences among breeds of domestic dogs (Canis lupus familiaris): current status of the science. Appl. Anim. Behav. Sci. 155, 12-27.

Montgomery, S.H. 2019. Are brain weights estimated from scaling relationships suitable for comparative studies of animal cognition? Anim. Cogn. 22, 1191-1195.

Pérez-Barbería, F.J., Shultz, S. and Dunbar, R.I.M. 2007. Evidence for coevolution of sociality and relative brain size in three orders of mammals. Evolution. 61, 2811-2821.

Richet, C. 1891. Poids du cerveau, de la rate et du foie, chez les chiens de differéntes tailles. In Comptes Rendus Des Séances de La Société de Biologie et de Ses Filiales. (Ed.), Masson. Paris, France: Masson, pp: 405-415.

Roth, G. and Dicke, U. 2005. Evolution of the brain and intelligence. Trends Cogn. Sci. 9, 250-257.

Sacher, G.A. and Staffeldt, E.F. 1974. Relation of gestation time to brain weight for placental mammals: implications for the theory of vertebrate growth on JSTOR. Am. Nat. 108, 593-615.

Sholl, D. 1948. The quantitative investigation of the vertebrate brain and the applicability of allometric formulae to its study. Proc. R. Soc. London. Ser. B - Biol. Sci. 135, 243-258.

Shultz, S. and Dunbar, R. 2010. Encephalization is not a universal macroevolutionary phenomenon in mammals but is associated with sociality. Proc. Natl. Acad. Sci. 107, 21582-21586.

Stephan, H. and Andy, O.J. 1969. Quantitative comparative neuroanatomy of primates: an attempt at a phylogenetic interpretation. Ann. N. Y. Acad. Sci. 167, 370-387. 